\title{
AN INVESTIGATION OF GENOTYPE ENVIRONMENT INTERACTIONS IN OAT LINES (AVENA SATIVA) DERIVED FROM COMPOSITE POPULATIONS
}

\author{
W. POWELL AND M. S. PHILLIPS \\ Scottish Crop Research Institute, Pentlandfield, Roslin, Midlothian, Scotland
}

Received 9.viii. 83

\section{SUMMARY}

An investigation of genotype environment interactions in two composite populations (A65 and A66) of spring oats grown at four contrasting sites in Scotland showed that:
(a) Genotype environment interactions were operative in both composite popu- lations. In the A65 composite population a significant portion of these interactions was accounted for by a linear function of the environmental means. In the A66 composite, however, most of the interaction was indepen- dent of this linear component and therefore the square root of the variance was used as a measure of environmental sensitivity.
(b) There was no significant correlation between mean grain yield and environ- mental sensitivity in the two composite populations examined.
(c) It was confirmed that the selection environment is very important in determining the environmental sensitivity of resultant selections.
(d) The implications of these results for the oat breeder are discussed.

\section{INTRODUCTION}

The occurrence of genotype environment interactions, which are usually revealed by an analysis of variance of trial data, presents the plant breeder with a number of problems. Firstly, he must decide whether to breed generally adaptable varieties or varieties adapted to particular subsets of environments and secondly, he must decide on a plan of action to select and evaluate his material with respect to its adaptability. The general procedure followed is to carry out early generation selection in one environment and then test elite genotypes for their adaptability in several environments. This procedure has formed the basis of varietal production at a number of breeding institutes. However, recent evidence would suggest that the environment in which selection is exercised is critical in determining the environmental sensitivity of selected lines. This finding has a strong theoretical basis and has been shown empirically to be the case in fungi (Jinks and Connolly, 1973, 1975), animals (Bateman, 1971; Orozco, 1976) and higher plants (Jinks and Pooni, 1982). Thus a knowledge of genotype environment interactions is essential for the design of an effective breeding programme and therefore investigations were carried out on genotype environment interactions in oat lines derived from composite cross populations.

\section{MATERIALS AND METHODS}

\section{(i) Oat composite cross populations}

The first composite population (A65) was produced by pollinating $\mathrm{F}_{2}$ plants obtained from a hybrid bulk population with pollen from plants 
representing the first 150 genotypes of an oat collection at Pentlandfield. The second composite (A66) was produced the following year by using $F_{2}$ plants from the $\mathrm{A} 65$ composite and pollinating with the next 150 genotypes from the collection. The $F_{1}$ and $F_{2}$ seed from each year's crossing were multiplied at Pentlandfield and then sub-divided into three sub-populations. These populations were then grown from, $F_{3}$ to $F_{6}$, without conscious selection, at one of three sites. The sites used were: Queenstonbank, East Lothian; Tullochgorum, Inverness-shire and Ledaig, Argyll. Forty-five lines, 15 from each of the three sites were selected after visual inspection for morphological characters and grown, together with seven controls, in a common trial at each of the three centres and at the Murrays Farm, East Lothian.

In addition to the composite population, data were also available on 14 inbred genotypes produced by conventional pedigree methods and grown in trials at six sites within the UK.

\section{(ii) Statistical analyses}

Both trials of the composite populations were randomised complete block designs with five replicates. The genotypic effects from the composite populations were regarded as being random. Although the trials were not grown at a random sample of the environments in which oats are grown in Scotland, "environments" and "blocks within environments" were regarded as random effects as the trials could not be repeated exactly in future years. Hence the following random effects model (Model II) was used for the analysis of variance.

$$
Y_{i j k}=\mu+G_{i}+E_{j}+G E_{i j}+B_{j(k)}+e_{i j(k)}
$$

where: $Y_{i j k}$ is the yield of the $i$ th genotype in the $k$ th block within the $j$ th environment = mean yield, $G_{i}=$ effect due to the $i$ th genotype, $E_{j}=$ effect due to the $j$ th environment, $G E_{i j}=$ effect due to the interaction of the $i$ th genotype with the $j$ th environment (genotype environment interaction), $B_{j(k)}=$ effect due to the $k$ th block in the $j$ th environment, $e_{i j(k)}=$ random sampling component.

The genotypes derived by pedigree breeding were grown in a randomised complete block design with three replicates. In this case the varieties were regarded as having fixed effects and the environments random. Therefore a mixed model was used for the analysis of variance. The components of variance $\sigma_{G}^{2}, \sigma_{E}^{2}, \sigma_{G E}^{2}, \sigma_{e}^{2}$ and $\sigma_{B}^{2}$ were calculated from the expected mean squares appropriate to the model.

Where the genotype environment interactions were significant, a joint regression analysis was carried out (Yates and Cochran, 1938) and the interactions partitioned into a heterogeneity of regression item and a deviation from regression item. The regression item was tested for significance against the deviation from regression item when the latter was significantly larger than the error item.

\section{RESUlts}

Analyses of variance for grain yield of the two oat composite populations and of the pedigree lines are given in table 1a. This shows that for all three 


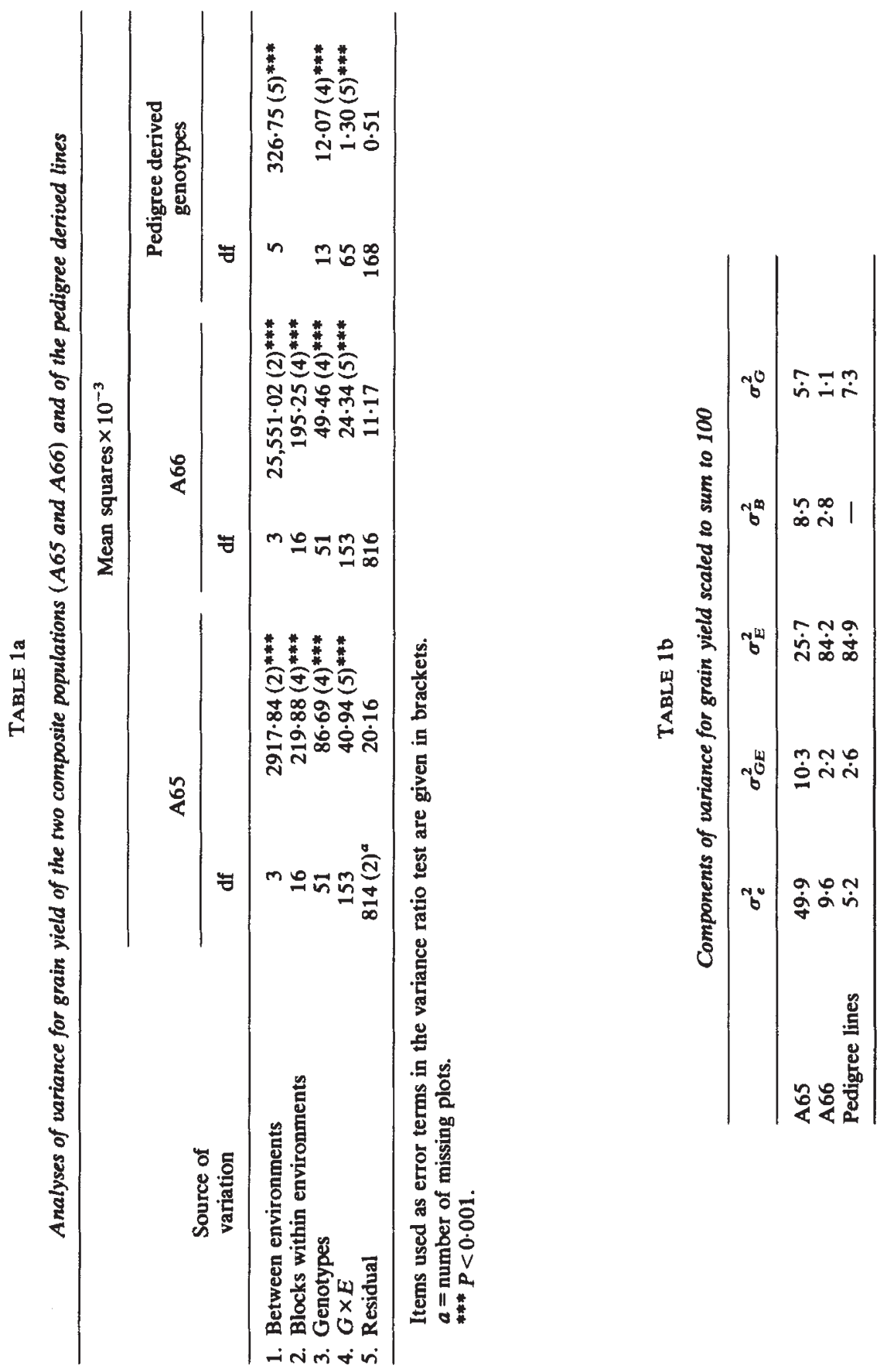


populations there were highly significant $(P<0.001)$ differences between genotypes and between environments as well as significant interactions of genotypes with environments. The estimates of the components of variance are shown in table $1 \mathrm{~b}$. In order to facilitate comparisons, the components have been scaled to sum to 100 . The main interest is the relative importance of the genetic $\left(\sigma_{G}^{2}\right)$, environmental $\left(\sigma_{E}^{2}\right)$ and genotype environment interaction $\left(\sigma_{G E}^{2}\right)$ components. In all cases the $\sigma_{G E}^{2}$ component was small compared with the $\sigma_{E}^{2}$ component. However, the $\sigma_{G E}^{2}$ component was larger than the $\sigma_{G}^{2}$ component in both the A65 and A66 oat composite populations. In contrast the $\sigma_{G}^{2}$ component for the pedigree lines was larger than the $\sigma_{G E}^{2}$ component.

Neither a logarithmic nor a square root transformation of the yield data was successful in removing the interaction, suggesting that it is not a scalar effect. This item was therefore analysed further by joint regression analysis (table $2 \mathrm{a}$ ). In the case of the A65 composite population the heterogeneity between regressions was highly significant $(P<0.001)$ when tested against the deviations from regression and therefore accounted for much of the interaction. Thus the linear model will have considerable predictive value for the genotypes concerned, though the model is not entirely satisfactory since a significant amount of the variation due to genotype environment interaction remains unaccounted for.

TABLE 2

Joint regression analyses for grain yield in oats

\begin{tabular}{lcccc}
\hline & \multicolumn{3}{c}{ Mean square $\left(\times 10^{-3}\right)$ and significance } \\
\cline { 2 - 5 } & $\begin{array}{c}\text { Genotypes } \times \\
\text { environments }\end{array}$ & $\begin{array}{c}\text { Heterogeneity of } \\
\text { regression } \dagger\end{array}$ & $\begin{array}{c}\text { Deviations from } \\
\text { regression }\end{array}$ & Error \\
\hline $\begin{array}{l}\text { A65 composite } \\
\text { population }\end{array}$ & $40 \cdot 94^{* * *}$ & $59 \cdot 10^{* * *}$ & $31 \cdot 86^{* * *}$ & $20 \cdot 16$ \\
$\begin{array}{l}\text { A66 composite } \\
\text { population }\end{array}$ & $24 \cdot 34^{* * *}$ & $21 \cdot 52$ N.S. & $25 \cdot 73^{* * *}$ & $11 \cdot 17$ \\
\begin{tabular}{l} 
Pedigree lines \\
\hline
\end{tabular} & $1 \cdot 30^{* * * *}$ & 1.85 N.S. & $1 \cdot 17^{* * * *}$ & 0.51 \\
\hline
\end{tabular}

$\dagger$ Tested against deviations from regressions.

N.S. $P>0.005$.

*** $P<0.001$.

In the case of the A66 population and the pedigree lines the heterogeneity of regression mean squares were not significant and therefore no simple linear relationship exists, in these cases, between genotype environment interaction and environmental values. Nevertheless it does not rule out the possibility that individual regressions for certain genotypes may be highly significant when tested against their mean square.

Since the linear regression did not account for all the genotype environment interaction it was considered inappropriate to use the slope as a measure of environmental sensitivity. Environmental sensitivity was thus measured as the square root of the variance for grain yield over the four sites. It has been widely reported that there is a positive correlation between yield and environmental sensitivity (Lawes, 1977). Indeed, data from the pedigree lines support this finding $(r=0.90)$. In contrast, the two composite 


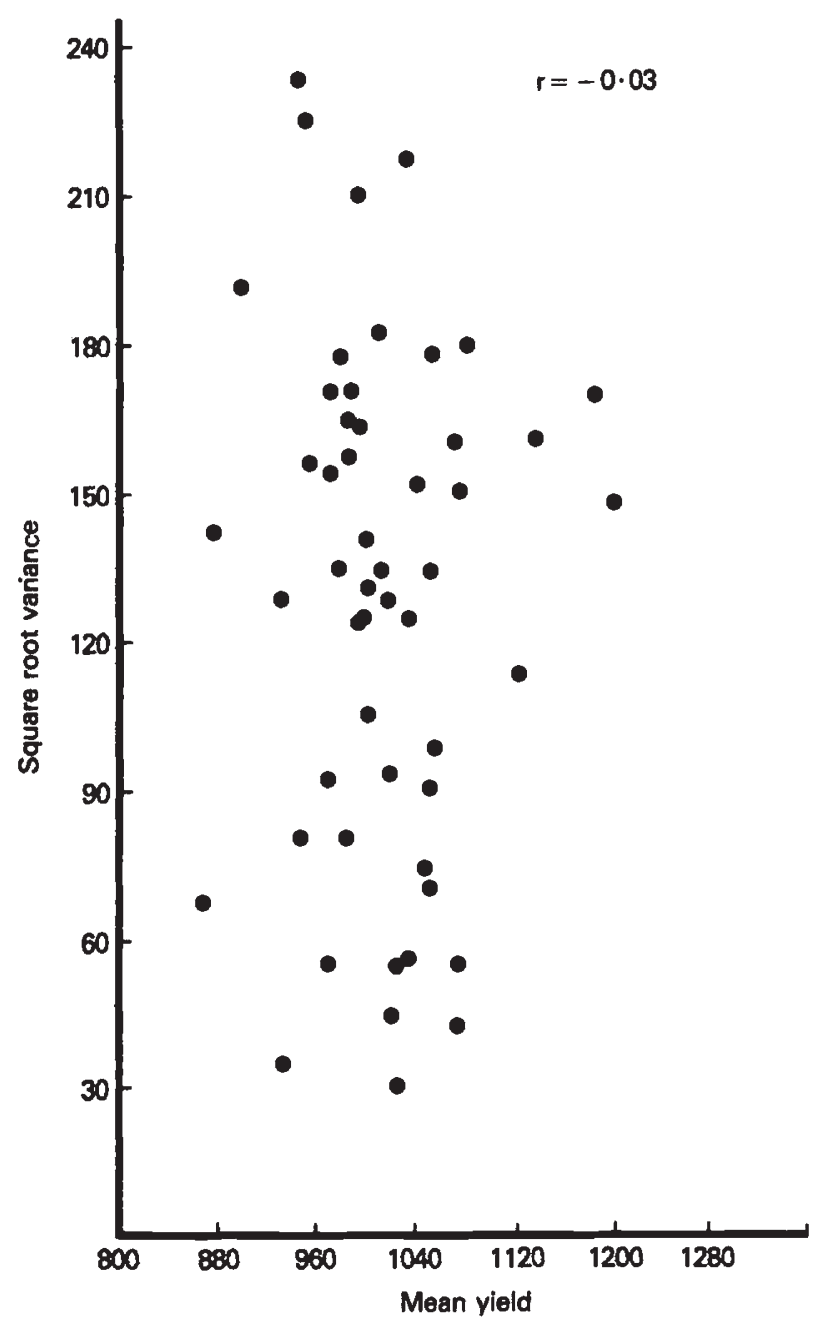

FIG. 1. Relationship between mean yield and Environmental Sensitivity (square root of variance) for the 52 genotypes included in the A65 composite population.

populations do not show this trend between high yield and environmental sensitivity. This is illustrated graphically in fig. 1 for the A65 composite, where it can be clearly seen that it is possible to select high yielding genotypes with low environmental sensitivities and vice versa.

The results of selection at different sites, as means and environmental sensitivity, are presented in table 3 . The mean performance is the average plant yield achieved by a selection in the four trials. Also included in table 3 are the high and low yielding selections that would have been made on the basis of average yield of the 52 genotypes at the four sites. In certain cases the same genotypes were identified at the different sites and therefore their mean yields will be the same. The results are similar to those reported by Jinks and Connolly (1975) and Jinks and Pooni (1982) who summarised their findings as follows: 


\section{TABLE 3}

Mean performance and environmental sensitivities of high and low selections from the A65 and A66 composite populations made in the poorest, best and in an average environment and on the basis of mean performance in all environments

\begin{tabular}{lccccc}
\hline & \multicolumn{2}{c}{ High selection } & & \multicolumn{2}{c}{ Low selection } \\
\cline { 2 - 3 } \cline { 5 - 6 } $\begin{array}{l}\text { Selection } \\
\text { environment }\end{array}$ & $\begin{array}{c}\text { Mean } \\
\text { performance }\end{array}$ & $\begin{array}{c}\text { Environmental } \\
\text { sensitivity }\end{array}$ & & $\begin{array}{c}\text { Mean } \\
\text { performance }\end{array}$ & $\begin{array}{c}\text { Environmental } \\
\text { sensitivity }\end{array}$ \\
\hline A65 & & & & & \\
Poorest & 1201.6 & 149.69 & & 900.3 & 192.62 \\
Average & 1201.6 & 149.69 & & 990.8 & 192.42 \\
Best & 1186.9 & 170.26 & & 379.3 & 143.98 \\
Mean of all & & & & & \\
Environments & 1201.6 & 149.69 & & 879.3 & 143.98 \\
A66 & & & & & \\
Poorest & 831.5 & 293.68 & & 767.0 & 337.71 \\
Average & 962.8 & 360.41 & & 695.8 & 238.24 \\
Best & 851.2 & 381.54 & & 695.8 & 238.24 \\
Mean of all & & & & \\
Environments & 962.8 & 360.41 & & 668.1 & 321.50 \\
\hline
\end{tabular}

(a) Selection for high yield in an above average environment leads to selections with above average environmental sensitivity.

(b) Selection for low yield in an above average environment leads to selections with below average environmental sensitivity.

(c) Selection for high yield in a below average environment will lead to selections with below average environmental sensitivity.

(d) Selection for low yield in a below average environment will lead to selections with above average environmental sensitivity.

These relationships between selection environment and environmental sensitivity are predictable from the theory formulated by Jinks and Connolly (1975). It should also be noted that it was possible to identify at Queenstonbank (average site) the genotype with the highest overall yield.

\section{Discussion}

Although the pedigree lines and composite populations were not grown in the same environments and the observations on them are therefore not strictly comparable, some general conclusions can be made. The segregating populations of the pedigree lines were grown and selected in a favourable agricultural environment, the Murrays. Reference to table 3 clearly indicates that selection at this site will maximise the environmental sensitivity of high selections and minimise that of low selections. It is therefore probable that parallel selection has occurred for high yielding and environmentally sensitive genotypes.

The composite cross populations were created by intercrossing a large number of genotypes and bulking the progeny. These genetically diverse populations were then multiplied and maintained for six generations of natural pollination with some ( 3.5 per cent) natural crossing (Phillips, 1978) 
and subjected to natural selection in the particular geographic areas of interest. Homozygous lines derived from the composite populations were then tested for adaptability at four sites which represent the range of environmental and agronomic conditions of importance.

The present results have a number of implications for the oat breeder given that under certain circumstances high environmental sensitivity and high mean yield may not be an optimal combination. Available evidence would suggest that recent selection carried out in high imput environments has produced environmentally sensitive genotypes (Lawes, 1977). Breeders must therefore acknowledge this fact when considering crossing programmes since most of the current varieties will be sensitive to environmental change. The composite cross populations described did produce a number of lines with different levels of environmental sensitivity and yield. However, the method is slow and dependent on recombination and natural selection and furthermore it is assumed that natural selection is operating in the direction favourable to the plant breeder.

An alternative approach may be to generate homozygous lines quickly by single seed descent (Brim, 1966). One could then monitor levels of environmental sensitivity as well as mean yield and simultaneously select for both characters. The level of work involved may make this too demanding and an equally effective method would be to select for mean yield in an environment which has been choosen to produce the desired level of environmental sensitivity. Selection of the highest yielding genotype at Queenstonbank produced the genotype with the highest mean yield over the four sites. Thus selection in one environment, Queenstonbank in this case, allowed identification of genotypes which would normally require testing over 4 sites.

Work carried out on experimental material such as Schizophyllum, Tribolium, mice and Nicotiana have shown that both high and low selections differed in their environmental sensitivities according to whether selection was based on assessment in a good or in a poor environment. These results have now been confirmed in an important agricultural crop, Avena sativa. Furthermore the association between high mean and variance or "scalar relationship" cannot explain the present findings since the low mean performance at the poor site of the selected genotype also has a high environmental sensitivity (table 3 ). The association between mean performance and environmental sensitivity is not therefore a statistical consequence, but the result of different genetic systems controlling these characters as has been previously demonstrated in Drosphila, (Caligari and Mather, 1975), Nicotiana (Jinks and Pooni, 1982) and Schizophyllum (Jinks and Connolly, 1975) and therefore amenable to manipulation by the oat breeder.

Acknowledgements. We would like to thank Mr D. Cameron and Dr W. T. B. Thomas for allowing us to use data from the spring oat breeding programme. Helpful discussions with Drs P. D. S. Caligari and J. E. Bradshaw are also gratefully acknowledged.

\section{REFERENCES}

BATEMAN, N. (1971). Selection of mice for growth on constant and on changing maize-milk diets. Anim. Prod. 13, 425-440.

BRIM, C. A. (1966). A modified Pedigree method of selection of Soyabeans. Crop. Sci. vol. 6, 1966, 220. 
CAligARI, P. D. S. AND MATHER, K. (1975). Genotype environment interaction: III Interactions in Drosophila melanogaster. Proc. R. Soc. Lond. B. 191, 387-411.

JINKS, J. L. AND CONNOLLY, V. (1973). Selection for specific and general response to environmental differences. Heredity, 30, 33-40.

JINKS, J. L. AND CONNOLLY, V. (1975). Determination of the environmental sensitivity of selection lines by the selection environments. Heredity, 34, 401-406.

JINKS. J. L. AND POONI, H. S. (1982). Determination of the environmental sensitivity of selection lines of Nicotiana rustica by the selection environments. Heredity, 49, 291-294.

LAWES, D. A. (1977). Yield improvement in Spring oats. J. Agric. Sci. Camb. 89, 751-757.

OROzCO, F. (1976). A dynamic study of genotype environment interaction with egg laying of Tribolium castaneum. Heredity, 39, 345-355.

PHILLIPS, M. S. (1978). The frequency of natural crossing in composite cross population of oats grown in Scotland. Cereal Research Communication Vol. 6, 1978. 67-69.

YATES, F. AND COCHRAN, W. G. (1938). The analysis of groups of experiments. J. Agric. Sci. $28,556-580$. 